\title{
Formalizing Knowledge in Multi-Scale Agent-Based Simulations
}

\author{
Endre Somogyi \\ Department of Computer \\ Science, \\ Biocomplexity Institute \\ Indiana University \\ Bloomington, IN 47405 \\ somogyie@indiana.edu
}

\author{
James P. Sluka \\ Department of Intelligent \\ Systems Engineering \\ Biocomplexity Institute \\ Indiana University \\ Bloomington, IN 47405 \\ jsluka@indiana.edu
}

\author{
James A. Glazier \\ Department of Intelligent \\ Systems Engineering \\ Biocomplexity Institute \\ Indiana University \\ Bloomington, IN 47405 \\ jaglazier@gmail.com
}

\begin{abstract}
Multi-scale, agent-based simulations of cellular and tissue biology are increasingly common. These simulations combine and integrate a range of components from different domains. Simulations continuously create, destroy and reorganize constituent elements causing their interactions to dynamically change. For example, the multi-cellular tissue development process coordinates molecular, cellular and tissue scale objects with biochemical, biomechanical, spatial and behavioral processes to form a dynamic network. Different domain specific languages can describe these components in isolation, but cannot describe their interactions. No current programming language is designed to represent in human readable and reusable form the domain specific knowledge contained in these components and interactions. We present a new hybrid programming language paradigm that naturally expresses the complex multi-scale objects and dynamic interactions in a unified way and allows domain knowledge to be captured, searched, formalized, extracted and reused.
\end{abstract}

\section{INTRODUCTION}

A computational model is an abstract representation of a physical system that captures relevant details. It also provides the user with the ability to retain the knowledge captured when such a model is built, allowing subsequent users to benefit from the shared knowledge inherent in the first model as they design their subsequent models.

An ontology is a formalized description of a particular view of reality which includes a precisely defined set of object types, a hierarchical taxonomy that organizes the objects into categories, a set of relationships that may apply between objects and a syntax needed to express the ontology's structure. Ontologies define a uniform naming convention and provide a way to define objects and interactions. However, they do not have the capability to define dynamics. A programming language is a formal computer language

Permission to make digital or hard copies of all or part of this work for personal or classroom use is granted without fee provided that copies are not made or distributed for profit or commercial advantage and that copies bear this notice and the full citation on the first page. Copyrights for components of this work owned by others than ACM must be honored. Abstracting with credit is permitted. To copy otherwise, or republish, to post on servers or to redistribute to lists, requires prior specific permission and/or a fee. Request permissions from permissions@ acm.org.

MODELS'16 Saint-Malo France

(C) 2016 ACM. ISBN 978-1-4503-2138-9.

DOI: http://dx.doi.org/10.1145/2976767.2976790 designed to encode semantic information in a form that is understandable to computers.

A computational model represents some underlying physical reality. For a model to be maximally useful, it must provide semantic meaning to both humans and computers. These semantics specify a set of mathematical and computational operations carried out by a program but do not have any intrinsic connection to the original physical phenomena that the model is meant to represent. Biological meaning can be assigned to the model's structures (but rarely is) by linking the computational implementation to ontologies that map to the original physical concepts.

A simulation environment to model dynamic and wideranging phenomena encountered in multi-scale agent-based biological simulations must have a modeling language that can express the requisite object types and their respective interactions (which we define as processes). Numerous approaches [10], including ours, separate the natural phenomena being modeled into two classes: objects and processes. Objects define the physical things being studied. Processes define the dynamics and interactions of and between objects. These objects and processes work together to define how a system evolves over time, i.e., they work together to produce a dynamical model.

\section{OBJECTIVES}

Capturing and building knowledge in the physical sciences is a gradual and iterative process. Often one begins with some vague notion that observed components somehow interact with each other. Perhaps one object is created by the other, perhaps one object somehow regulates or influences the other. These are qualitative observations, and serve as a valuable foundation or staging area for further investigation.

Agent based models of biological phenomena enable us to better understand how function and organization arise out of the interactions of constituent objects and processes. Building such models involves creating and assembling many complex components in order to understand the larger phenomenon 18. A biological modeling language must provide language constructs - the basic building blocks that comprise models - that correspond directly to the building blocks found in biology. These building blocks enable users to build models out of virtual representations of biological objects and processes. Therefore, a modeling language approach must also enable users to encode and formalize biological concepts with language constructs that closely match 
physical reality. Biological models should form a repository where domain knowledge can be captured, stored, searched and extracted.

A key objective for a biological modeling language is that models must retain a description of the system being modelled within the model itself. In some domains outside of biology, the nomenclature of the domain is sufficiently well defined so that models in those systems describe both the targeted physical system as well as its computational implementation. For example, in electrical engineering the schematic description of a circuit and the simulation of the behavior of that circuit are clear and concise in part because there are a limited number of basic electrical devices (resistors, transistors, etc.) with which to model a circuit. In the biological domain the number of possible "parts" is much greater (and much more poorly defined) and the nomenclature of those parts is often ambiguous. Therefore, the biologist must use reference ontologies to define the biological concepts contained in his model, preferably using techniques contained within the model code that allow for the inclusion of the biological ontology-based description of the system being modeled. Unfortunately, the majority of current multiscale agent based models in biology have completely uncoupled the biological description of the system being modelled from the computational instantiation of that model. We believe it is critically important to develop technologies that allow for the description of both the biology being modeled and the particular numerical and computational instantiation of that model.

A biological modelling language should support qualitative and iterative model development. One typically begins formalizing biological knowledge by creating a qualitative description of empirical observations and gradually refining it over time as more information becomes available. A modeling language should therefore allow researchers to record incomplete observations using "placeholder" objects and processes. Say a researcher observes a particular cell type undergoing apoptosis in the presence of a particular signaling molecule but has no knowledge of the details of this process. The researcher should be able to create "placeholder" elements (cell type, signaling molecule, apoptosis process) and save them by establishing a high-level model whose details can be refined over time.

In order to have predictive power, a simulator should read a biological model and compile it into an executable model that can be run and analyzed. The results of the simulation can be used to make experimentally testable predictions. We are developing a biological modeling language methodology called the Continuous Conditional Object Process Methodology (CCOPM) to address these objectives. In turn, these objectives support the ultimate goal of capturing, storing, sharing and enabling the search for and re-use of knowledge acquired as users model biological phenomena. We have designed this modeling language approach to extend an existing object-oriented language. Extending an established language allows us to re-use and extend existing infrastructure such as language parsers and syntax trees rather than developing them de novo every time we encounter a new model.

\section{RELATED WORK}

We explored many programming language paradigms, including functional, procedural, object-, logic-, and equation- oriented approaches in creating a language to describe cellular and tissue biology. While none of these languages had the expressive capacity to capture and formalize biological knowledge, they offered many ideas which we incorporated into our formalism.

Alan Kay, one of the pioneers of object oriented programming had cell-to-cell signaling in mind as a motivating example for the object-oriented approach, "I thought of objects being like biological cells and/or individual computers on a network, only able to communicate with messages" [15]. In object-oriented languages, a program consists of objects that communicate with one another through discrete messages. Actor oriented languages [14] are similar to object-oriented languages except that each object (actor) runs concurrently communicates with other actors via messages. Actors may send messages, create or destroy other actors, and modify local state in response to messages. However, in biological simulations, communication tends to be continuous rather than discrete. Here, inter-agent communication is mediated by the agent's environment since chemical signals secreted by one agent must advect and diffuse through the environment to arrive at other agents.

Cyber-physical systems (CPS) integrate discrete computer control with the real-world physical environment. In CPS, embedded digital computers monitor and control physical devices, often with feedback loops. CPS are an example of a hybrid system, which combines discrete and continous dynamics. Engineers frequently model [5] CPS with equationoriented languages such as Modelica [19], The Math Work's Simulink or National Instrument's LabVIEW. Models enable engineers to perform extensive analysis, formal verification [2] and simulation of designs in order to evaluate their correctness and performance. Hybrid system models are similar to agent-based biological models in that that they combine discrete, event triggered procedural logic with classic differential-equations that define continuously evolving physical quantities. Engineering problems however tend to be easier to define than those found in multi-scale agentbased biological simulations. The number of components, their identities, and their connectivity - which components interact with what other components - typically remain fixed for the duration of most simulations. Equation-oriented languages excel at defining components and their interconnections and interactions, but have limited support for the re-arrangement, addition or removal of components. Equationoriented languages also tend to not be spatially aware in that they do have ways of describing spatial concepts such as spatial regions, motion, deformation, or chemical diffusion, all of which are fundamental to biology.

A number of domain specific languages (DSL) can describe general agent-based simulations of interacting autonomous decision-making discrete entities called agents [3]. These DSLs are well-suited for describing discrete agent-agent communication via messages or method invokation, and encoding agent responses to messages, however they also tend to lack intrinsic language constructs to describe spatial concepts, and typically have no way of describing continous dynamics with differential equations.

Interchange formats such as Systems Biology Markup Language [13] or Cell Markup Language [17] can encode and exchange chemical network models or ordinary differential equation models. Interchange formats however typically cannot describe spatial or structural rearrangement, nor dynam- 
ically add or remove components. Interchange formats are rarely Turing Complete, meaning they cannot be used to describe arbitrary biological processes.

Few languages seem to exist that reference ontologies in order to connect computational constructs to physical meaning. A reference to ontologies allows the construction of models that convey physical meaning as well as the necessary computational structure.

A language for biological agent based modeling must integrate 1) creation, deletion, dynamic rearrangement, and messaging capacity of actor-oriented languages, 2) description of continous dynamics via differential equations and event handling found in equation-oriented languages, 3) description of mechanical forces and fields found in molecular dynamics simulators, 4) reference biological ontologies to convey physical meaning. Our approach integrates these concepts into a single programming language approach.

\section{APPROACH}

\subsection{Formalizing Biological Phenomena}

The first step in formalizing knowledge is writing it down in such a way that it has semantic meaning for both humans and computers. In order to specify biological phenomena, we need a programing language that has language constructs corresponding directly to natural phenomena.

We are developing the Continuous Conditional Object Process Methodology (CCOPM), a programming language approach to augment existing object-oriented languages, with a set of constructs that can describe the features present in multi-scale agent-based models. Biological phenomena can be naturally represented using two key concepts: objects and processes. Objects represent the "things" such as molecules, proteins, cells, fluids, or materials. An object is defined as any instantiable physical or logical entity that has certain state-full or structural properties. Processes represent anything that causes a change in state. A process may, for example, represent a transformation in which substrates are consumed and products are produced, or it may alter the state of an object, either continuously or discretely. Unlike traditional programming languages, processes in the CCOPM can operate concurrently and continuously. This paradigm of representing the world in terms of objects and processes forms the basis of the Cellular Behavior Ontology (CBO) 21, and also the Object Process Methodology (OPM) 6].

Some examples of this object/process formalism applied to biological phenomena are 1) chemical kinetics: molecules are defined as objects, reactions are defined as processes. A process may consume multiple substrates and produce multiple products; 2) cellular mitosis: the mitosis process consumes a cell and produces two cells; 3) cellular chemical uptake: the process acts on a cell type, consumes external chemical quantities and produces internal chemical quantities.

Ontological identifiers defined by the $\mathrm{CBO}$ and other bioontologies can be attached to any language element (objects or process) which allows us to group these elements by their biological roles such apoptosis, mitosis, transport, molecular processes and movement processes, phenotypic change, etc. Ontological identifiers enable us to retain biological knowledge.

Objects are the nouns, the actual things being described.
Objects are such things as molecules, cells, membranes, ion channels, the extra-cellular matrix, fluids, etc. Objects have quantifiable characteristics such as location, amount, concentration, mass and volume. Objects define a state; they are comparable to standard data structures in conventional programming languages. Objects may inherit and extend other objects, or may contain other objects. Objects are grouped into two categories: continuous and discrete. Continous objects describe things such as continuously valued chemical or molecular fields which have position-dependent concentrations. Chemical fields may be 3D in that they are bounded by a surface or $2 \mathrm{D}$ in that they exist on a surface. For reasons of numerical efficiency, users may specify fields as spatially uniform.

Processes are the verbs. Processes may create and destroy objects, alter the state of objects, transform objects, or consume and produce sets of objects. As in nature, multiple CCOPM processes act concurrently on objects and may act at different rates, but may only be active under certain circumstances. Processes may be continuously active, or may be explicitly triggered by specific conditions or invoked directly by some other process. Processes may also be combined or aggregated, such that a process may be hierarchically composed of child processes, and child processes may be ordered either concurrently or sequentially. Processes fall into two categories: continuous and discrete.

A set of conditions may be attached to any process definition. Conditions are specified in a when clause, much as they are in Modelica. Conditions operate differently for discrete processes than they do for continuous processes. Discrete processes are very similar to SBML events, in that a discrete process is triggered only when its condition expression transitions from false to true. The discrete process will also trigger at any future time when the condition expression makes this transition. Conditions on a continous process determine when the process should be active.

Discrete processes are similar to functions in functional languages in that they are a sequence of statements. The runtime triggers discrete processes when their condition is met. Discrete processes can consume, create or modify discrete objects. For example, users may define a discrete creation process which creates new cell instances in a spatial region, or a discrete deletion process which deletes a cell instance in response to some condition. The runtime manages a pool of threads which execute triggered processes. The runtime also continuously monitors each discrete process condition expression, and when the condition evaluates to true, the runtime places the triggered process into a priority queue and the next available thread executes the triggered processes.

Continuous processes ( $\kappa$-processes) operate on continuous valued objects, and can be thought of as a generalization of the concept of chemical reactions. $\kappa$-processes consume reactants and produce products. Continuous processes must define a rate function which defines how fast the transformation (reaction) is occurring. The arguments to a $\kappa$-process must be labeled as either a reactant or a modifier, and a $\kappa$-process yields a set of zero or more products. An unlimited number of continuous $\kappa$-processes can act on an object instance, and the rate of change of this object instance is defined as the stoichiometric sum of all the currently active transformation processes that are consuming or producing this object. $\kappa$-processes may be used to define chemical reac- 
tions in a spatial compartment (such as cells) or membrane transport.

Rate processes ( $\rho$-processes) use a rate function to define the rate of change of a set of arguments. Only one $\rho$-process may be active on an object at a time; $\rho$-processes and $\kappa$ processes are mutually exclusive.

Force processes ( $\phi$-processes) provide a way to describe spatial change, such as motion, deformation, adhesion or response to external forces. $\phi$-processes are similar to force functions in molecular dynamics. A $\phi$-process can be defined to act on one or between two spatial objects, hence a $\phi$-process may have one or two arguments, and both of them must be spatial object subtypes. $\phi$-processes return the force that acts on its arguments. Any motion processes (adhesion, taxis, deformation) can be specified via a suitable force process. For example, when an adhesion process is active between a surfaces of a pair of cells, the adhesion process applies a force between the cell surfaces at the locations where the surfaces are in contact. This adhesive force acts to keep the cells in contact and resists surface separation.

The language runtime automatically applies the force functions to spatial objects and calculates the net force acting on each spatial object. The runtime then calculates the time evolution of each spatial object, typically as $\mathbf{v} \propto \mathbf{F} / m$, where velocity is proportional to the net force acting on each spatial object.

Types serve to classify variable instances into categories. The type of a variable determines the kind of data that may be stored in that variable. The type of an object defines what operations are valid on instances of that object type, i.e., we can sum two numeric types, but adding a numeric type to a string is ill-defined. Most programming languages do not have a concept related to the biological notion of a phenotype. A phenotype in biology is a metric, an observable categorization that determines a cell's type. A phenotype is defined by a set of rules or conditions such that when these conditions are met, we say that a cell is of such type.

The CCOPM extends the basic concept of dynamic or static types with a rule-based type, which is related to the concept of typestate oriented programming 24]. Here, the type may be defined via a set of rules, and when all of those rules are met, we say that a variable instance is a certain type. This notion is important because biological cells frequently undergo phenotypic change, that is, they change type over time. Processes are defined to operate on a specific type, and the runtime automatically applies these processes to all instances of the specified type. Here we can create a type definition based on a set of conditions; when all of these conditions are met, the type expression becomes true, and the processes corresponding to that type definition now automatically become active on all object instances for which the type evaluates to true.

Scope Resolution determines how symbol names resolve to a value. Programming languages typically have either static or dynamic scoping 11. Component composition in agent-based tissue simulations poses challenges that are not commonly encountered in traditional programming languages. Here, variables may also carry a spatial extent, for example, a chemical concentration exists over a region of space. So, whenever a chemical of a certain name is read or written, the value depends on where in space the read/write operation is occurring. Hence, the scope resolution in a spatial environment is related to the underlying spatial configuration. Furthermore, multiple different chemical networks may be placed inside of a cell or other spatial region. If this region is defined as a well-stirred compartment, all of the networks operate in the same space. Hence, any chemical species that these networks operate on must be connected to the same species in all of the other networks within that space. Additionally, chemical species may transfer across physical boundaries (e.g., cell membranes) that exist between distinct spatial regions.

In order to account for the spatial nature of objects, we introduce a new scope resolution rule which we call spatial scoping. Spatial scoping extends the traditional dynamic scoping with environmental acquisition 9]. Environmental acquisition was originally used in graphical user interface design to enable hierarchical composition of user interface widgets. In spatial scoping, scoping blocks correspond to a spatial region. In dynamic scoping, non-local symbols resolve to the scoping block where the function was called. In spatial scoping, non-local symbols resolve to the spatial region where the function is evaluated. This concept applies uniformly to spatially extended components such as chemical fields, as well as objects with well-defined boundaries such as cells. For example, a chemical network could exist inside of a cell. Here, each instance of that cell type will likely have different chemical values. The values that the chemical network processes read resolves to the local values found in each cell. Similarly, say we add a transport process to model an ion channel to a cell's surface, and this transport process's rate function defines a symbol corresponding to a chemical field. Even as the cell moves, the symbol always resolves to the value of the chemical field that corresponds to the cell surface location.

\subsubsection{Visual Model Definition}

Diagrams are nearly always the first step in capturing knowledge. A scan through a textbook or journal in the life sciences will quickly reveal that knowledge is nearly always presented as diagrams and accompanying text. Usability is one of the biggest impediments to adoption of formal languages by biomedical researchers 4]. Diagrams are a way to summarize a mechanistic understanding of a set of observations, a way to informally or qualitatively present information. Unfortunately, as intuitive as diagrams may be, they contain no semantic information that is understandable by computers, and contain tacit knowledge only for humans. Diagrams are essentially free-form. Although significant efforts have been made to standardize a graphical notation for biochemical and regulatory pathways (http://www.sbgn.org), little progress has been made in most biological domains. Most diagrams continue to be made qualitatively, and can be highly ambiguous without annotation. For example, the $\rightarrow$ symbol used extensively in diagrams can mean influencing, moving towards, becoming, consuming, or something else entirely.

Process Modeling Languages lend themselves well to visual representation 8. In order to create a direct manipulation user interface that enables visual display and editing of models, we extend the algorithms we have previously used to graphically render and edit the MathML language 20 22 . The model source is read into an abstract syntax tree (AST), which is treated as a live document much like an HTML DOM in that it may be edited and manipulated and 
will be re-drawn in real time. As a user visually edits the document and the user interface converts the user's actions into operations which restructure the document AST.

All graphical elements are rendered using the current standard for systems biology, SBGN. This standard is already used in many applications, including the widely used CellDesigner 7], a diagram editor for drawing gene regulatory and biochemical networks.

A prototype of the user interface is shown in fig. 1].

\subsection{Model Compilation and Execution}

Capturing and formalizing existing biological knowledge is the first step in expanding our understanding of biological phenomena. Once biological knowledge has been formalized in the form of a biological model, the compiler can compile the biological model into an executable model. An executable model enables users to simulate the time evolution of biological models and make quantitative predictions that can help guide future experiments and expand our understanding.

The set of processes in CCOPM can be thought of as a heterogenous hybrid system [16, where continuous processes define a set of continuous time differential equations which interact with the event triggered discrete processes. The continuous processes define a set of equations that specify a relationship between quantities and how they evolve over time. The spatial processes (discrete object motion, continous field diffusion) extend the classical notion of hybrid system.

Discrete process semantics are identical to conventional programming languages, and the compiler can compile them using established techniques 11. Conditions define when a discrete process should be invoked. Condition triggered discrete process semantics are identical to SBML. A condition triggers a discrete process whenever the condition expression transitions from false to true. Continous processes define the time evolution of the continous variables, such as chemical concentrations. Continous processes form a reaction network. We have previously developed techniques to compile continous process, chemical reaction networks, and discrete events into executable code 23. Force functions define the motion of spatial objects. The force function also define a set of equations that describe the time evolution of spatial objects. The compiler generates code that sums all of the forces on every spatial object such that the time evolution of every spatial object is defined by a friction dominated Langevin equation, $\gamma \frac{d \mathbf{x}}{d t}=\mathbf{F}+\xi(t)$, where $\mathbf{x}$ is position, $\gamma$ is the friction parameter, $\mathbf{F}$ is the net force, and $\xi(t)$ is the stochastic force at temperature $T$.

Time Evolution is how objects' states change over time. Time evolution can be thought of as an application of the classical propagator, $\Gamma(t+\delta t)=e^{i \mathcal{L} \delta t} \Gamma(t)$, to advance the simulation forward in time by $\delta t$. Our formalism defines essentially three kinds of dynamics: discrete, continuous, and spatial. These dynamics have distinct time scales: discrete processes execute instantaneously in terms of simulation time; continuous and spatial processes occur over continuous time, with continuous processes typically operating on a faster time scale than spatial processes. Spatial state variables (those that participate in the spatial evolution process) typically change much more slowly than other variables. In the interest of numerical efficiency, time slicing is used to solve the time evolution of the system. Each time step consists of three stages. Stage 1 applies the spatial evolution process, which numerically calculates the time evolution of the spatial variables via a Langevin equation and performs spatial diffusion of chemical fields. Stage 2 checks conditions to determine which discrete process to trigger and which continuous process to apply. Finally, Stage 3 invokes a numeric integrator to calculate the time evolution of the continuous variables.

\subsection{Integrating Knowledge}

The programming language approach developed here enables biological knowledge to be formalized such that it has meaning for both humans and computers. The visual model definition enables biologists without any computer science training to build biological models. A compiler compiles biological models into executable code which can yield predictive, quantitative results. Together, these form a complete system for capturing and creating biological knowledge. The model is the central repository of knowledge depicted in figure 1. The model combines the dynamics and ontological descriptors stored as an AST and a database of state values. A parser reads source code documents and can download ontological descriptions from a variety of internet based sources such as http://www.bioontology.org The model also participates in a standard model-view-controller triad where users may view and interact with it. Finally, the model is also connected to a simulation engine. This simulator reads and compiles the the syntax tree and reads a current state configuration from the model. The simulator then advances this state forward in time and records these subsequent states back into the model state database. Users may extract a variety of output types from the model, such as LaTeX documents suitable for integration into publications or a variety of output data intended for handoff to analysis packages.

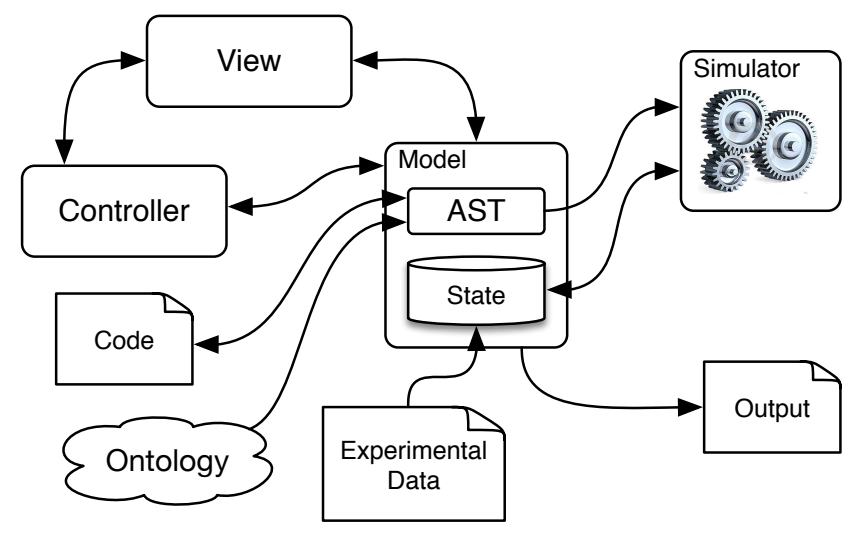

Figure 1: A complete system for capturing and representing domain knowledge, the model is the central repository of knowledge.

\section{RESULTS}

We have created a prototype implementation of the CCOPM called Cayman, which is based on the Microsoft TypeScript language. We have implemented a prototype source-to-source translator that compiles Cayman to a combination of conventional Python, SBML and XML. The generated XML and Python code are specific to the CompuCell3D 25] CPM 
based multi-cell simulator. Future versions will compile to other simulation platforms or directly to machine executable code.

This section will discuss diagrammatically building a biological model from a user perspective and will describe the model builder GUI's responses to user actions. Here we first summarize epithelial cell extrusion as presented in [12, then discuss how this biological knowledge is formalized and captured with the Cayman language.

A layer of cells called an epithelium surrounds the internal and external surfaces of organs in most animals. The epithelium is a polarized layer of cells consisting of an outer layer and several lower layers. It forms a barrier that is constantly exposed to damage, yet must be selectively permeable to specific compounds. Epithelial cells have one of the highest turnover rates in organisms; dead, damaged or dying cells are continually extruded while preserving the barrier. Like most cells, individual epithelial cells require a nonhomogeneous membrane in order to function correctly 1]. Their plasma membranes are often organized into several discrete regions, each having specific attributes and carrying out different tasks. Such non-symmetric cells are called "polarized cells". Epithelial cells have distinct apical, lateral and basal membrane regions. Each of these regions contain different sets of trans-membrane proteins and channels. Epithelial cells adhere to neighboring cells via the E-cadherin proteins expressed in the cell's lateral domain. E-cadherin proteins stick to other like E-cadherin proteins. The E-cadherins are normally located near the lateral-apical boundary in a ring shape. This organization causes epithelial cells to stick more more tightly to their neighbors at the apical face. The exact mechanism which organizes these cadherins into this ring shape is not known, but it is likely some active process, or possibly the organization arises out of contact with other epithelial cells. It is not known if isolated epithelial cells have such a cadherin distribution, nor if contact with other epithelial cells induces or contributes to the cadherin distribution. The basal surface of the epithelium adheres to the underlying basal-laminar surface via integrin trans-membrane proteins present on the basal surface that stick to the basal-laminar surface.

Epithelial cells are continually exposed to damage and frequently replaced. A biological extrusion processes normally forces damaged epithelial cells out of the apical face of the epithelium. Cells can initiate this process by secreting S1P signaling lipids into their surrounding region. Neighboring cells sense $\mathrm{S} 1 \mathrm{P}$ when it binds to their $\mathrm{S}_{1} \mathrm{P}_{2}$ receptors. When a S1P lipid binds to the the external domain of the $\mathrm{S}_{1} \mathrm{P}_{2}$ receptor, they form a complex, and the receptor undergoes a conformational change to become active. Most of the signaling molecules discussed here can be in either active or inactive conformations. The internal domain of active $\mathrm{S}_{2} \mathrm{P}_{2}$ activates a p115RhoGEF protein. Active p115RhoGEF reacts with inactive Rho proteins, turning the Rho active Rho proteins, and resetting the RhoGEF to inactive. Active Rho proteins can react with myosin fibers by reorienting and attaching the fibers to locations on the cell's basal surface. The myosin fibers then cause the basal and basal lateral surfaces to contract. The contraction of basal-lateral surfaces causes the cell to become conically shaped and thereby allows the neighboring cells to force the extruded cell outwards.

The cell extrusion process, like many biological processes, is complex but resilient. A malfunctioning extrusion process can force cells out of the basal surface, which can lead to many problems. Currently it is not clear how sensitive the epithelial extrusion process is to adhesion between neighboring epithelial cells or between epithelial cells and the underlying basal lamina. A biological model and simulation would allow biologists to virtually explore the effects of altering adhesion, which could give insight into developing future experiments.

The first step in creating a biological model is identifying the relevant objects and which processes act on them. Here we identify a single cell type, the epithelial cell, this cell has a membrane with three distinct regions. All cells in the Cayman environment are derived from a built-in Cell type, which in turn is a subtype of SpatialRegion. These are bounded, fully enclosed three-dimensional regions of space. Here, we develop a model using a diagrammatic representation, where each element has a cartoon representation as in Figure 2. We first drag and drop a cell type on a the blank canvas. The controller interprets this action by adding a new subtype of Cell to the model defintion, and we may then edit various attributtes, much like the GUI builder in Microsoft's Visual Studio or Apple's XCode do.

The biological mechanism which forms and organizes the three different membrane regions is not known, but none the less, some biological process must exist which is responsible for the observed experimental evidence. In order to define a multi-region membrane, we may add MembraneRegion objects to our new cell type's membrane by visually dragging a pictorial representation of these objects onto the cell membrane. The controller will again interpret the user actions and modifies the underlying AST by adding an appropriate MembraneRegion definition to the cell definitions's membrane collection.

The series of chemical reactions described above form part of a signaling cascade, a series of chemical reactions which ultimately result in a cell response to external stimuli. Signaling cascades are usually set in motion in response to a signaling event, such as binding to the $\mathrm{S}_{1} \mathrm{P}_{2}$ receptor. At this point, we do not have enough information to specify reaction rate equations for these chemical reactions. However, we may guess that the reaction rate obeys mass-action kinetics, or we may specify that a reaction does occur, but leave the reaction rate equation blank. Ignoring the reaction rate, we can readily write down the above biological descriptions as a list of chemical reactions, where the active state of a compound is denoted with an "a" sub-script: 1) $\mathrm{S} 1 \mathrm{P}+\mathrm{S}_{2} \longrightarrow$ $\left.\left.\mathrm{S} \mathrm{P}_{2 \mathrm{a}}, 2\right) \mathrm{S} 1 \mathrm{P} \longrightarrow \emptyset, 3\right) \mathrm{S}_{2} \mathrm{P}_{2 \mathrm{a}}+\mathrm{RhoGEF} \longrightarrow \mathrm{S}_{2} \mathrm{P}_{2}+$ $\mathrm{S} 1 \mathrm{P}+\mathrm{RhoGEF}_{\mathrm{a}}$, 4) RhoGEF $\mathrm{a}+\mathrm{Rho} \longrightarrow \mathrm{RhoGEF}_{\mathrm{a}} \mathrm{Rho}_{\mathrm{a}}$. In actuality, the active and inactive states of a molecule are not separate chemical species, however they currently have to be represented as separate chemical species as Cayman does not yet support multi-state chemical species. We may add these chemical equations to our model using either a textual or graphical notation. For example, we may write the first two of these reaction equations using the textual notion (based on TypeScript) as

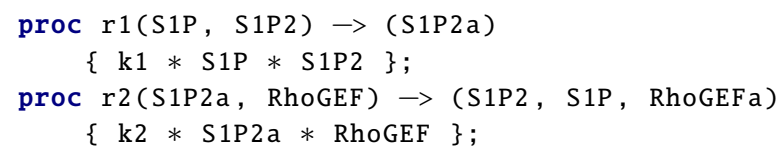

where the body of the process defines the rate of reaction. These are examples of a continous process, where the argu- 
ments and return types are continuous types. The compiler assembles all continuous process definitions into a system of differential equations which a numerical integrator solves runtime. The rate of change of the value of each continuous variable instance is the stoichiometric weighted sum of all the participating reaction rates. The above pair of continuous processes for example define the rate of change of each S1P instance as $d / d t(S 1 P a)=(k 1 * S 1 P * S 1 P 2)-$ $(k 2 * S 1 P 2 a *$ RhoGEF $)$ More details of compiling chemical equations into executable simulations can be found in our chemical reaction JIT compiler 23. We may also write this chemical signaling network diagrammatically, where the user has a similar experience to applications such as CellDesigner. These four reaction equations are also presented diagrammatically in Figure 2 .

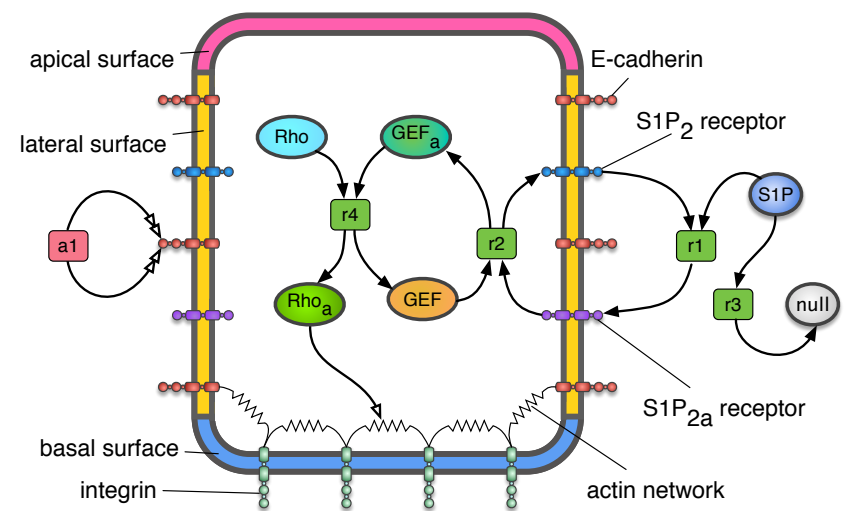

Figure 2: Diagrammatic representation and editing of the epithelial extrusion model. According to SBGN standard, closed arrows, $\rightarrow$, represent consumption and production, and open arrows, $\rightarrow$, represent modifiers. We have introduced the double open arrow, $\rightarrow$, to represent force processes.

Epithelial cells adhere to one another using E-cadherin proteins and respond to $\mathrm{S} 1 \mathrm{P}$ signals with the $\mathrm{S}_{1} \mathrm{P}_{2}$ receptors, both of which are located on their lateral surfaces. We may add this protein and receptor to our cell model by dragging a surface adhesion molecule and a receptor onto our cell's lateral surface region. Because the $\mathrm{S}_{2} \mathrm{P}_{2}$ receptor can exist in one of two states, we have two receptors, one for each state that the receptor can be in. Then we can finish adding the remaining signaling molecules to our diagram and connect them together in a signaling network. We can then add an adhesion process to the E-cadherin protein, and parameterize this process with an appropriate contact adhesion force values. When the model is compiled and executed, the runtime will apply a force between lateral surfaces when they are in contact. This force will tend to keep the surfaces in contact and resist separation. We can model the effect of the actin network by adding a spring network to the basal surface. In order to respond to the $\mathrm{Rho}_{\mathrm{a}}$ signal, we can write the functional form of the spring network to calculate the force using the local concentration of $\mathrm{Rho}_{\mathrm{a}}$ as a parameter. Gudipaty and Rosenblatt 12 state that the actin network causes the basal surface to contract. We can model this observation with a contractile force acting on each pair of basal surface vertices,

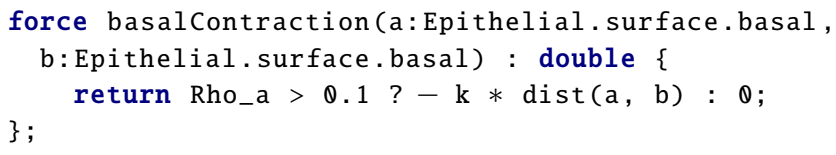

The compiler recognizes that Epithelial.surface.basal is a collection type, thus treats it as a vector much as does MATLAB. The Rho_a symbol is not defined in the local scope, so the compiler searches in the next level up spatial scoping block, which happens to be the cell's cytosol. Thus the Rho_a resolves to the concentration value of the of this variable. This function is defined as a two-body force, so it calculates the magnitude of the force between two vertices, and the compiler generates code that applies this force to each pair of arguments in the appropriate direction. The runtime sums all of the forces on each vertex and uses this information to determine where to advect the vertices in the spatial evolution phase of the time step. Once our epithelial cell type definiton is complete, we can replicate it to create an initial configuration for the epithelial layer.

The model building process captures the biological knowledge embodied in published results and experiments. This knowledge is in a form that can be easily mined, reused and extended by others, accelerating knowledge accumulation. If the model has sufficient quantitative details such as rate equations and parameters, the compiler can compile it into an executable model in order to perform simulations. A biologist could then perform a series of virtual experiments to determine how sensitive the epithelial extrusion process is to adhesion between neighboring epithelial cells. The results of these virtual experiments yield information such as which parameter ranges correspond to observed behavior. This new knowledge could then be used to guide future physical experiments.

\section{CONCLUSIONS}

No programming language approach currently exists - neither commercial, nor open-source - that is capable of describing multi-scale agent-based systems interacting with a complex physical environment in a single, unified way. The majority of multi-scale agent based models written today have completely uncoupled the underlying physical description of the system being modelled from the computational instantiation of that model. The CCOPM introduced in this paper combines the expressivity required for representing these complex physical concepts and their interactions with an existing general purpose language that maintains a connection to the original underlying physical system. The CCOPM enables us to create a platform which can capture and formalize domain knowledge such that it may be searched, extracted and reused.

\section{ACKOWLEDGEMENT}

We would like to thank Dr. John Scott Gens and Dr. Marie Gingras for their insight and valuable conversations. We acknowledge generous financial support from the Falk Medical Research Trust Catalyst Program, National Institutes of Health, National Institute of General Medical Sciences, National Institute of Environmental Health Sciences and National Institute of Biomedical Imaging and Bioengineering, grants U01 GM111243, R01 GM076692 and R01 GM077138 and an Indiana University Cooperative Research grant. 


\section{REFERENCES}

[1] B. Alberts, A. Johnson, J. Lewis, M. Raff, K. Roberts, and P. Walter. Molecular Biology of the Cell. Garland Science, 4th edition, 2002.

[2] R. Alur. Formal verification of hybrid systems. In Proceedings of the International Conference on Embedded Software EMSOFT, pages 273-278, New York, Oct. 2011. ACM.

[3] R. H. Bordini, L. Braubach, M. Dastani, and A. Seghrouchni. A survey of programming languages and platforms for multi-agent systems. Informatica, 30:33-44, 2006.

[4] L. Cardelli and C. Priami. Visualization in Process Algebra Models of Biological Systems. In S. Tansley and K. M. Tolle, editors, The Fourth Paradigm Data-intensive Scientific Discovery. research.microsoft.com, 2009.

[5] P. Derler, E. A. Lee, and A. S. Vincentelli. Modeling cyber-physical systems. In Proceedings of the IEEE, pages 13-28, 2012.

[6] D. Dori. Object-Process Methodology: A Holistic Systems Paradigm. Springer, Berlin, 2002.

[7] A. Funahashi, M. Morohashi, H. Kitano, and N. Tanimura. CellDesigner: a process diagram editor for gene-regulatory and biochemical networks. Biosilico, 1:159-162, 2003.

[8] M. Gambini. The Design of Graphical Process Modeling Languages: from Free Composition to Modular Construction. PhD thesis, Università degli Studi di Verona, Verona, Sept. 2012.

[9] J. Gil and D. H. Lorenz. Environmental acquisition: a new inheritance-like abstraction mechanism. $A C M$ SIGPLAN Notices, 31:214-231, 1996.

[10] P. Grenon and B. Smith. SNAP and SPAN: Towards Dynamic Spatial Ontology. Spatial Cognition 85 Computation, 4:69-104, Mar. 2004.

[11] D. Grune, K. Van Reeuwijk, H. E. Bal, C. J. Jacobs, and K. Langendoen. Modern Compiler Design. Springer-Verlag, New York, 2012.

[12] S. A. Gudipaty and J. Rosenblatt. Epithelial cell extrusion: Pathways and pathologies. Seminars in Cell $\&$ Developmental Biology, May 2016.

[13] M. Hucka, A. Finney, H. M. Sauro, H. Bolouri, J. C. Doyle, H. Kitano, , the rest of the SBML Forum, A. P. Arkin, B. J. Bornstein, D. Bray, A. Cornish-Bowden, A. A. Cuellar, S. Dronov, E. D. Gilles, M. Ginkel, V. Gor, I. I. Goryanin, W. J. Hedley, T. C. Hodgman, J. H. Hofmeyr, P. J. Hunter, N. S. Juty, J. L. Kasberger, A. Kremling, U. Kummer, N. Le Novere, L. M. Loew, D. Lucio, P. Mendes, E. Minch, E. D. Mjolsness, Y. Nakayama, M. R. Nelson, P. F. Nielsen, T. Sakurada, J. C. Schaff, B. E. Shapiro, T. S. Shimizu, H. D. Spence, J. Stelling, K. Takahashi, M. Tomita, J. Wagner, and J. Wang. The systems biology markup language (SBML): a medium for representation and exchange of biochemical network models. Bioinformatics, 19:524-531, Mar. 2003.

[14] R. K. Karmani and G. Agha. Actors. In Encyclopedia of Parallel Computing, pages 1-11. Springer US, Boston, MA, 2011.

[15] A. C. Kay. Alan Kay on the Meaning of "Object-Oriented Programming". Accessed Mar. 30,
2016 ,

http://www.purl.org/stefan_ram/pub/doc_kay_oop_en 2003.

[16] E. A. Lee and H. Zheng. Operational semantics of hybrid systems. In International Workshop on Hybrid Systems Computation and Control, pages 25-53. Springer, Hybrid Systems: Computation and Control, 2005.

[17] C. M. Lloyd, M. D. B. Halstead, and P. F. Nielsen. CellML: its future, present and past. Progress in Biophysics and Molecular Biology, 85:433-450, June 2004.

[18] D. N. Macklin, N. A. Ruggero, and M. W. Covert. The future of whole-cell modeling. Current opinion in biotechnology, 28:111-115, 2014.

[19] S. E. Mattsson, H. Elmqvist, and M. Otter. Physical system modeling with Modelica. Control Engineering Practice, 6:501-510, Apr. 1998.

[20] L. Padovani. MathML Formatting. PhD thesis, Univeristy of Bologna, Bologna, Jan. 2003.

[21] J. P. Sluka, A. Shirinifard, M. Swat, A. Cosmanescu, R. W. Heiland, and J. A. Glazier. The cell behavior ontology: describing the intrinsic biological behaviors of real and model cells seen as active agents. Bioinformatics, 30:2367-2374, 2014.

[22] E. Somogyi. gnumerator mathml rendering library. Accessed Mar. 30, 2016, http://numerator.sourceforge.net, 2005.

[23] E. T. Somogyi. Simulation of electrochemical and stochastic systems using just in time compiled declarative languages. $\mathrm{PhD}$ thesis, Indiana University, Bloomington, Dec. 2014.

[24] R. E. Strom and S. Yemini. Typestate: A Programming Language Concept for Enhancing Software Reliability. In IEEE Transactions on Software Engineering, pages 157-171, 1986.

[25] M. H. Swat, G. L. Thomas, J. M. Belmonte, A. Shirinifard, D. Hmeljak, and J. A. Glazier. Multi-Scale Modeling of Tissues Using CompuCell3D. Methods in Cell Biology, 110:325-366, 2012. 